\title{
Reference station weighting and frame optimality in minimally constrained networks
}

\author{
C. Kotsakis \\ Department of Geodesy and Surveying \\ Aristotle University of Thessaloniki, Thessaloniki 54124, Greece \\ Email: kotsaki@topo.auth.gr
}

\begin{abstract}
The aim of this paper is to present a general solution of the weight choice problem for the reference stations in minimally constrained network adjustment. Our treatment is based on the optimization of the accuracy of the estimated network coordinates over all possible choices of minimum constraints on the reference stations. The optimal criterion considers the joint effect of the data and datum noise on the estimated coordinates and it is implemented over an arbitrary subset of the network stations. The final solution leads to a flexible treatment of the datum choice problem by allowing the weight matrix of the reference stations to be tuned to various options regarding the frame quality in the adjusted network.
\end{abstract}

Keywords. Network adjustment; minimum constraints; datum choice problem; data noise effect; datum noise effect; frame optimization.

\section{Introduction}

The datum choice problem (DCP) is a well known hassle in geodetic network adjustment with coordinate-based models. It is linked to the optimal estimation of a set of coordinates from a singular system of normal equations obtained by the network data analysis within a linearized least squares (LS) framework (e.g. Dermanis 1985; Schaffrin 1985; Teunissen 1985). The usual treatment of the DCP requires that a set of external constraints is used to complement the missing datum information in the available data. Several options exist for the selection and the implementation of those constraints into the network adjustment procedure, each of which has its own merits for the final estimated solution. In this study we concentrate on the socalled minimum constraints (MCs) which fix the datum defect of the geodetic network without interfering with its estimable characteristics from the available data (Sillard and Boucher 2001).

These datum constraints are typically applied over a number of reference stations that are included in the network adjustment and have a priori known coordinates with respect to the user's desired reference frame. Their general expression is given in terms of the linear system
$\mathbf{E}\left(\mathbf{x}-\mathbf{x}^{\mathrm{ext}}\right)=\mathbf{0}$

which corresponds to the well known inner constraints or, more precisely, partial inner constraints since only a part of the network stations is involved in the datum definition process (Meissl 1969; Blaha 1971). The above constraints enforce the harmonization of the (non-estimable) frame parameters of the geodetic network with the respective frame parameters implied by the known coordinates $\mathbf{x}^{\text {ext }}$ of the reference stations. The matrix $\mathbf{E}$ stems from the usual Helmert transformation model using only the rows that correspond to the datum defect of the underlying network (Sillard and Boucher 2001).

The use of Eq. (1) offers a restrictive optimality for the estimated coordinates in the chosen frame. In fact, the fundamental property of the network solution under these constraints is the minimization of the propagated data noise on the estimated coordinates of the reference stations. This suggests that there are two limitations which Eq. (1) is not able to handle in network adjustment problems, namely:

(i) the optimal control of the propagated data noise on other network stations (apart from the reference stations), and

(ii) the optimal control of the random errors in the a priori reference coordinates $\mathbf{x}^{\text {ext }}$ and their propagated effect (hereafter called datum noise effect) on other network stations.

Both of these issues are crucial in the context of the optimal datum choice for minimally constrained networks and they have to be treated in a more general setting than the one provided by the classic inner constraints in Eq. (1). Specifically, the use of a weight matrix for the reference stations according to the extended form of inner constraints

$\mathbf{E P}\left(\mathbf{x}-\mathbf{x}^{\text {ext }}\right)=\mathbf{0}$

enables us to overcome the aforementioned limitations within a zero-order optimization scheme for geodetic networks. This result has been established in Kotsakis (2013) where the choice problem for the weight matrix $\mathbf{P}$ was tackled on the basis of the 
joint minimization of the data and datum noise effects over all network stations.

The aim of this paper is to present a useful extension of the previous result by considering the minimization of the data/datum noise effects over an arbitrary subset of the network stations. This generalization provides a flexible treatment of the DCP by allowing the weight matrix of the reference stations to be tuned to various options regarding the frame quality of the adjusted network. Essentially, we formulate herein an MC-based scheme for geodetic network adjustment under an optimality principle for the estimated coordinates of any desired group of the network stations.

\section{Problem formulation}

The general problem that is treated herein can be briefly described as follows. Our starting point is a singular system of normal equations (NEQ)

$$
\mathbf{N}\left(\mathbf{X}-\mathbf{X}^{\mathbf{0}}\right)=\mathbf{u}
$$

which is obtained from the linearized LS adjustment of a geodetic network. It is considered that the rank defect of the above system is solely caused by the datum deficiency in the used data. Without loss of generality, we assume that any nuisance parameters have been eliminated beforehand from the NEQ system, so that the term $\mathbf{X}-\mathbf{X}^{\mathbf{O}}$ contains only the unknown corrections to the approximate coordinates of the network stations.

The total coordinate vector in Eq. (3) is partitioned as

$$
\mathbf{X}=\left[\begin{array}{l}
\mathbf{x} \\
\mathbf{x}^{\prime}
\end{array}\right]
$$

where $\mathbf{x}$ refers to the reference stations that are included in the network and $\mathbf{x}^{\prime}$ corresponds to the new stations whose coordinates represent the primary unknowns of the estimation problem at hand.

The rationale of our study relies on the exact (MC-based) inversion of the NEQ system using the datum information that is contained in the reference stations. In such cases the estimated coordinates $\hat{\mathbf{x}}^{\prime}$ produce a "soft" densification of the external frame (i.e. the one realized by the prior coordinates of the reference stations) in the sense that the datum transfer through the MCs does not interfere with framerelated parameters that are already defined by the available data.

According to the previous setting, the usual datum choice is provided by the system of (partial) inner constraints in Eq. (1). The corresponding solution is obtained through the general formula (Koch 1999)

$$
\hat{\mathbf{X}}=\mathbf{X}^{\mathrm{o}}+\left(\mathbf{N}+\mathbf{H}^{\mathrm{T}} \mathbf{H}\right)^{-1}\left(\mathbf{u}+\mathbf{H}^{\mathrm{T}} \mathbf{c}\right)
$$

where the constraint matrix $\mathbf{H}$ and the vector $\mathbf{c}$ are given by the following expressions

$$
\begin{aligned}
& \mathbf{H}=\left[\begin{array}{ll}
\mathbf{E} & \mathbf{0}
\end{array}\right] \\
& \mathbf{c}=\mathbf{E}\left(\mathbf{x}^{\mathrm{ext}}-\mathbf{x}^{\mathrm{o}}\right)
\end{aligned}
$$

The covariance $(\mathrm{CV})$ matrix of the above solution has the form (ibid.)

$$
\begin{aligned}
\boldsymbol{\Sigma}_{\hat{\mathbf{X}}} & =\left(\mathbf{N}+\mathbf{H}^{\mathrm{T}} \mathbf{H}\right)^{-1} \mathbf{N}\left(\mathbf{N}+\mathbf{H}^{\mathrm{T}} \mathbf{H}\right)^{-1} \\
& =\left[\begin{array}{c:c}
\boldsymbol{\Sigma}_{\hat{\mathbf{x}}} & \boldsymbol{\Sigma}_{\hat{\mathbf{x}} \hat{\mathbf{x}}^{\prime}} \\
\hdashline \boldsymbol{\Sigma}_{\hat{\mathbf{x}} \hat{\mathbf{x}}} & \boldsymbol{\Sigma}_{\hat{\mathbf{x}}^{\prime}}
\end{array}\right]=\mathbf{N}^{-}
\end{aligned}
$$

and it corresponds to a generalized inverse of the normal matrix with minimum trace over the reference stations ${ }^{1}$. This is a well known result in network optimization theory upon which the use of inner constraints was introduced in geodetic practice (Blaha 1971); see also Grafarend (1974) and Schmitt (1982).

The CV matrix from Eq. (8) reflects only the data noise effect in the estimated coordinates, thus ignoring the influence of random errors in the known coordinates $\mathbf{x}^{\text {ext }}$ of the reference stations. The latter introduce a datum-related noise in the estimated solution which reflects the uncertainty of the (non-estimable part of the) coordinate system itself for the adjusted network. The optimal control of this datum noise effect, concurrently with the data noise effect, is an important issue for the minimally constrained network adjustment, however it cannot be handled through the classic inner constraints.

An additional concern stems from the fact that the minimum-trace property of the previous $\mathrm{CV}$ matrix refers only to its part related to the reference stations. The coordinates of the new stations are not estimated in an optimal way under the choice of Eq. (1). A worthy enhancement, therefore, is to look for an $\mathrm{MC}$ matrix $\mathbf{Q}$ to replace the classic inner-

\footnotetext{
${ }^{1}$ The minimum-trace property of the error CV submatrix $\boldsymbol{\Sigma}_{\hat{\mathbf{x}}}$ in Eq. (8) is equivalent to a simple (unweighted) LS fit of the adjusted network to the known coordinates $\mathbf{x}^{\text {ext }}$ of the reference stations using the Helmert transformation model that involves only the non-estimable frame parameters of the underlying network.
} 
constraint matrix E, so that the revised datum constraints

$\mathbf{Q}\left(\mathbf{x}-\mathbf{x}^{\text {ext }}\right)=\mathbf{0}$

yield optimal accuracy for the estimated coordinates at the new stations (or any selected subset of network stations) with respect to the desired reference frame.

A similar version of the above problem was presented in Kotsakis (2013) for the case of the joint minimization of the data/datum noise effects over all network stations. The theoretical investigation in that study showed that the MC matrix should have the factorized form $\mathbf{Q}=\mathbf{E P}$, with $\mathbf{P}$ being a suitable weight matrix for the reference stations.

Herein we treat the case of minimizing the data/datum noise effects over an arbitrary subset of the network stations. The optimal MC matrix should again have the same factorized form while the weight matrix of the reference stations will have a more general structure than the one given in Kotsakis (2013).

\section{General expressions for the CV matrix of a MC solution}

Before we proceed with the optimal datum choice in minimally constrained networks (more specifically, the optimal choice of the weight matrix for the reference stations), it is instructive to review the various $\mathrm{CV}$ matrices involved in the accuracy assessment of the estimated network coordinates.

In general, the total $\mathrm{CV}$ matrix of a MC solution can be expressed as a sum of two components

$$
\Sigma_{\hat{\mathbf{X}}}^{\text {total }}=\Sigma_{\hat{\mathbf{X}}}^{\mathrm{obs}}+\boldsymbol{\Sigma}_{\hat{\mathbf{X}}}^{\mathrm{mc}}
$$

which contain the contributions from separate error sources, that is the data and datum noise effects, respectively. Their analytic forms are derived through straightforward covariance propagation to Eq. (5) and they are given by the general expressions

$$
\boldsymbol{\Sigma}_{\hat{\mathbf{X}}}^{\mathrm{obs}}=\left(\mathbf{N}+\mathbf{H}^{\mathrm{T}} \mathbf{H}\right)^{-1} \mathbf{N}\left(\mathbf{N}+\mathbf{H}^{\mathrm{T}} \mathbf{H}\right)^{-1}
$$

and

$$
\boldsymbol{\Sigma}_{\hat{\mathbf{X}}}^{\mathrm{mc}}=\left(\mathbf{N}+\mathbf{H}^{\mathrm{T}} \mathbf{H}\right)^{-1} \mathbf{H}^{\mathrm{T}} \boldsymbol{\Sigma}_{\mathbf{c}} \mathbf{H}\left(\mathbf{N}+\mathbf{H}^{\mathrm{T}} \mathbf{H}\right)^{-1}
$$

The previous equations are valid for any network solution that is determined by an arbitrary set of MCs

$$
\mathbf{H}\left(\mathbf{X}-\mathbf{X}^{\mathbf{O}}\right)=\mathbf{c}
$$

where the pseudo-observation vector $\mathbf{c}$ is associated with a prior $\mathrm{CV}$ matrix $\boldsymbol{\Sigma}_{\mathbf{c}}$.

Taking into account well known algebraic identities from the MC theory in singular NEQ systems (e.g. Kotsakis 2012), the following equivalent expressions can be also used

$\boldsymbol{\Sigma}_{\hat{\mathbf{X}}}^{\mathrm{obs}}=\left(\mathbf{N}+\mathbf{H}^{\mathrm{T}} \mathbf{H}\right)^{-1}-\tilde{\mathbf{E}}^{\mathrm{T}}\left(\mathbf{H} \tilde{\mathbf{E}}^{\mathrm{T}}\right)^{-1}\left(\tilde{\mathbf{E}} \mathbf{H}^{\mathrm{T}}\right)^{-1} \tilde{\mathbf{E}}$

and

$\boldsymbol{\Sigma}_{\hat{\mathbf{X}}}^{\mathrm{mc}}=\tilde{\mathbf{E}}^{\mathrm{T}}\left(\mathbf{H} \tilde{\mathbf{E}}^{\mathrm{T}}\right)^{-1} \boldsymbol{\Sigma}_{\mathbf{c}}\left(\tilde{\mathbf{E}} \mathbf{H}^{\mathrm{T}}\right)^{-1} \tilde{\mathbf{E}}$

where $\tilde{\mathbf{E}}$ denotes the inner-constraint matrix for the entire network which, in accordance to the partition of Eq. (4), is expressed as

$\tilde{\mathbf{E}}=\left[\begin{array}{ll}\mathbf{E} & \mathbf{E}^{\prime}\end{array}\right]$ and $\mathbf{N} \tilde{\mathbf{E}}^{\mathrm{T}}=\mathbf{0}$

For more details and the mathematical proofs of the preceding equations see Kotsakis (2012, 2013). Note that, for simplicity, the a priori variance factor is assumed to be equal to one.

The CV matrix $\Sigma_{\hat{\mathbf{x}}}^{\text {obs }}$ is always singular and it contains the effect of the data noise on the MC solution. It corresponds to a reflexive generalized inverse of the normal matrix $\mathbf{N}$ and its rank defect is equal to the datum defect of the network observational model. The trace minimization of this matrix was used as a criterion for solving the DCP in the context of network optimization theory (e.g. Blaha 1971, Schmitt 1982), thus leading to the classic type of inner constraints for geodetic network adjustment.

The CV matrix $\Sigma_{\hat{\mathbf{x}}}^{\mathrm{mc}}$ is also singular and it reflects the datum noise effect in the MC solution. In fact, it quantifies the accuracy of the estimated coordinates due to random errors in the pseudoobservation vector c. From a geodetic viewpoint, it is a necessary component for the realistic accuracy assessment of frame realizations obtained via minimally constrained networks on a number of reference stations. In such cases Eq. (13) should be expressed in the partitioned form 


$$
\underbrace{\left[\begin{array}{ll}
\mathbf{Q} & \mathbf{0}
\end{array}\right]}_{\mathbf{H}} \underbrace{\left[\begin{array}{c}
\mathbf{x}-\mathbf{x}^{\mathrm{o}} \\
\mathbf{x}^{\prime}-\mathbf{x}^{\prime \mathbf{o}}
\end{array}\right]}_{\mathbf{X}-\mathbf{X}^{\mathrm{o}}}=\underbrace{\mathbf{Q}\left(\mathbf{x}^{\mathrm{ext}}-\mathbf{x}^{\mathrm{o}}\right)}_{\mathbf{c}}
$$

or equivalently

$$
\mathbf{Q}\left(\mathbf{x}-\mathbf{x}^{\mathrm{ext}}\right)=\mathbf{0}
$$

where $\mathbf{Q}$ is an arbitrary MC matrix to be applied to the reference stations of the underlying network (and it will be optimally determined in the next section).

Note that the vector $\mathbf{c}$ is often set to zero by selecting the approximate coordinates of the reference stations to be equal to their a priori known values in the desired frame. This does not eliminate the datum noise effect which should be always accounted in terms of the matrix $\Sigma_{\hat{\mathbf{X}}}^{\mathrm{mc}}$ (see Eq. (12) or (15)) using the auxiliary covariance expression

$$
\boldsymbol{\Sigma}_{\mathbf{c}}=\mathbf{Q} \boldsymbol{\Sigma}_{\mathbf{x}}^{\mathrm{ext}} \mathbf{Q}^{\mathrm{T}}
$$

where $\Sigma_{\mathbf{x}}^{\text {ext }}$ corresponds to the prior $\mathrm{CV}$ matrix of the reference station coordinates $\mathbf{x}^{\mathrm{ext}}$.

\section{Optimal datum choice in MC networks - a general formulation}

For the purpose of this study, the optimal datum choice is linked to the minimization of an objective functional that quantifies the accuracy of the estimated coordinates at (all or part of) the network stations. A standard option for this functional is the trace of the total CV matrix $\Sigma_{\hat{\mathbf{X}}}^{\text {total }}$ which was analytically described in the previous section. Hence, the DCP is formulated in terms of the optimization problem

$$
\min _{\mathbf{Q}} \operatorname{tr}\left(\mathbf{S} \Sigma_{\hat{\mathbf{X}}}^{\text {total }} \mathbf{S}^{\mathrm{T}}\right)
$$

or, more explicitly

$$
\min _{\mathbf{Q}} \operatorname{tr}\left(\mathbf{S} \boldsymbol{\Sigma}_{\hat{\mathbf{X}}}^{\mathrm{obs}} \mathbf{S}^{\mathrm{T}}+\mathbf{S} \boldsymbol{\Sigma}_{\hat{\mathbf{X}}}^{\mathrm{mc}} \mathbf{S}^{\mathrm{T}}\right)
$$

where $\mathbf{Q}$ is the sought MC matrix and $\mathbf{S}$ corresponds to a "selection matrix" for the participating stations in the optimality principle. Note that the
MCs are applied only to the reference stations (see Eq. (17)) while the optimality principle may refer to any subset of network stations.

Considering the partition scheme in Eq. (4), some examples of the selection matrix are

$$
\mathbf{S}=\left[\begin{array}{ll}
\mathbf{I} & \mathbf{0}
\end{array}\right], \quad \mathbf{S}=\left[\begin{array}{ll}
\mathbf{0} & \mathbf{I}
\end{array}\right], \quad \mathbf{S}=\mathbf{I}
$$

which can be used for the accuracy optimization of the estimated coordinates at the reference stations, at the new stations, or at all network stations, respectively.

The MC matrix that satisfies the optimality principle in Eq. (20) can be derived from the equation

$$
\frac{\partial \operatorname{tr}\left(\mathbf{S} \boldsymbol{\Sigma}_{\hat{\mathbf{X}}}^{\text {obs }} \mathbf{S}^{\mathrm{T}}\right)}{\partial \mathbf{Q}}+\frac{\partial \operatorname{tr}\left(\mathbf{S} \boldsymbol{\Sigma}_{\hat{\mathbf{X}}}^{\mathrm{mc}} \mathbf{S}^{\mathrm{T}}\right)}{\partial \mathbf{Q}}=\mathbf{0}
$$

The dependence of $\boldsymbol{\Sigma}_{\hat{\mathbf{X}}}^{\mathrm{obs}}$ and $\boldsymbol{\Sigma}_{\hat{\mathbf{X}}}^{\mathrm{mc}}$ on the matrix $\mathbf{Q}$ stems from Eqs. (14)-(15) taking also into account the relationships in Eqs. (17)-(18). After some lengthy derivations the solution of the last equation is obtained as

$\mathbf{Q}=\mathbf{E}\left(\boldsymbol{\Sigma}+\boldsymbol{\Sigma}_{\mathbf{x}}^{\mathrm{ext}}\right)^{-1}$

where the matrix $\boldsymbol{\Sigma}$ is defined by the formula

$$
\left(\mathbf{N}+\mathbf{S}^{\mathrm{T}} \mathbf{S} \tilde{\mathbf{E}}^{\mathrm{T}} \tilde{\mathbf{E}} \mathbf{S}^{\mathrm{T}} \mathbf{S}\right)^{-1}=\left[\begin{array}{c:c}
\boldsymbol{\Sigma} & \mathbf{L} \\
\hdashline \mathbf{L}^{\mathrm{T}} & \boldsymbol{\Sigma}^{\prime}
\end{array}\right]
$$

(the above partitioning is compatible with the one introduced in Eq. (4)). The proof of the above result for the case $\mathbf{S}=\mathbf{I}$ is given in Kotsakis (2013) whereas the proof for an arbitrary selection matrix $\mathbf{S} \neq \mathbf{I}$ can easily be obtained as a straightforward extension of the derivations given in that paper.

\section{General remarks}

The optimal weight matrix for the reference stations in $\mathrm{MC}$ network adjustment has the general form

$$
\mathbf{P}=\left(\boldsymbol{\Sigma}+\boldsymbol{\Sigma}_{\mathbf{x}}^{\mathrm{ext}}\right)^{-1}
$$

where $\Sigma_{\mathbf{x}}^{\text {ext }}$ is the CV matrix of their prior coordinates and $\boldsymbol{\Sigma}$ is an auxiliary matrix obtained by Eq. (23). We underline that the last expression stems from a formal optimization scheme which has led to 
the factorized form of Eq. (22), thus proving that the weighted inner constraints is indeed the appropriate tool to ensure special optimal properties for the realized frame in a minimally constrained network.

The weight matrix $\mathbf{P}$ depends on two components each of which has a distinct role in the MC network adjustment. The first component is responsible for minimizing the propagated data noise on the estimated coordinates of a group of network stations that is specified by the selection matrix $\mathbf{S}$. The second component, on the other hand, is related to the filtering of the random errors in the reference stations coordinates from the final network solution. This dual role of the weight matrix is dictated by the joint presence of the data and datum noise effects, both of which influence in their own way the frame quality in the adjusted network.

\section{Minimization of the data noise effect}

If we ignore the random errors in the reference stations coordinates (i.e. $\boldsymbol{\Sigma}_{\mathbf{x}}^{\text {ext }}=\mathbf{0}$ ) then the weighted MCs take the form

$\mathbf{E} \boldsymbol{\Sigma}^{-1}\left(\mathbf{x}-\mathbf{x}^{\text {ext }}\right)=\mathbf{0}$

and the resulting network solution will have the minimum data noise effect at the stations specified by the selection matrix $\mathbf{S}$ which is hidden in the weight matrix $\Sigma^{-1}$; see Eq. (23).

If the selection matrix involves only the reference stations of the underlying network, that is $\mathbf{S}=\left[\begin{array}{ll}\mathbf{I} & \mathbf{0}\end{array}\right]$, then it can be shown that Eq. (25) is reduced to the form

$$
\mathbf{E} \mathbf{E}^{\mathrm{T}} \mathbf{E}\left(\mathbf{x}-\mathbf{x}^{\mathrm{ext}}\right)=\mathbf{0}
$$

which, due to the invertibility of the matrix $\mathbf{E E}^{\mathrm{T}}$, is equivalent to

$$
\mathbf{E}\left(\mathbf{x}-\mathbf{x}^{\text {ext }}\right)=\mathbf{0}
$$

In this special case, therefore, we reproduce the classic (unweighted) inner constraints whose optimality is solely related to the minimization of the data noise effect at the reference stations.

\section{Minimization of the datum noise effect}

If we consider the minimization of the datum noise in the MC solution without accounting for the data noise effect, that is

$$
\min _{\mathbf{Q}} \operatorname{tr}\left(\mathbf{S} \Sigma_{\hat{\mathbf{X}}}^{\mathrm{mc}} \mathbf{S}^{\mathrm{T}}\right)
$$

then the datum choice will be provided by the weighted MCs

$$
\mathbf{E}\left(\Sigma_{\mathbf{x}}^{\text {ext }}\right)^{-1}\left(\mathbf{x}-\mathbf{x}^{\text {ext }}\right)=\mathbf{0}
$$

It is noted that the weight matrix of the reference stations is independent of the selected stations that participate in the optimality principle. In contrast to the data noise effect, the minimization of the datum noise effect, over all or part of the network, requires a fixed weighting of the reference stations in terms of their prior CV matrix. In fact, if we take into account Eqs. (15)-(18) then we obtain the covariance decomposition formulae

$$
\boldsymbol{\Sigma}_{\hat{\mathbf{X}}}^{\mathrm{mc}}=\tilde{\mathbf{E}}^{\mathrm{T}} \boldsymbol{\Sigma}_{\boldsymbol{\theta}} \tilde{\mathbf{E}}
$$

and

$$
\boldsymbol{\Sigma}_{\boldsymbol{\theta}}=\left(\mathbf{Q} \mathbf{E}^{\mathrm{T}}\right)^{-1} \mathbf{Q} \boldsymbol{\Sigma}_{\mathbf{x}}^{\mathrm{ext}} \mathbf{Q}^{\mathrm{T}}\left(\mathbf{E} \mathbf{Q}^{\mathrm{T}}\right)^{-1}
$$

where the matrix $\boldsymbol{\Sigma}_{\boldsymbol{\theta}}$ describes the accuracy with which the (non-estimable) frame parameters are defined in the minimally constrained network. The datum noise minimization is equivalent to the trace minimization of $\boldsymbol{\Sigma}_{\boldsymbol{\theta}}$ (see Kotsakis 2013) and it will not be affected by the selection matrix $\mathbf{S}$ that appears in Eq. (28).

\section{Conclusions}

The weight choice problem of the reference stations in minimally constrained networks has been investigated in this paper. Our treatment is based on the optimization of the total accuracy (considering both the data and datum noise effects) of the estimated coordinates over all possible choices of MCs on the reference stations. As a result of this procedure, we obtained a weighted type of MCs which encompasses the classic (unweighted) inner constraints as a special option within a more general setting for the datum choice problem. 
In contrast to Kotsakis (2013) the current treatment allows the accuracy optimization of the minimally constrained solution over an arbitrary subset of network stations and not necessarily over the entire network. Hence, the weight matrix of the reference stations is not generally unique since it depends on the network stations that participate in the optimality principle of Eq. (20). It is noted that the use of (the inverse of) the prior CV matrix of the reference stations as a weight matrix is warranted only for minimizing the datum noise effect in the estimated network coordinates - it does not contribute to the optimal control of the data noise effect over all or part of the network.

A useful extension of the present study is the treatment of the weight choice problem for the reference stations in the case of non-minimal datum constraints. In our current approach the matrix $\mathbf{E}$ refers only to the non-estimable frame parameters and the implementation of the datum constraints $\mathbf{Q}\left(\mathbf{x}-\mathbf{x}^{\text {ext }}\right)=\mathbf{0}$ does not affect any estimable frame characteristics in the underlying network. The case where the constraint matrix $\mathbf{Q}$ refers also to estimable frame parameters (e.g. scale in the case of GNSS networks) is more complicated since several properties that have been used in this paper's algebraic derivations will simply not hold true.

\section{References}

Blaha G (1971) Inner adjustment constraints with emphasis on range observations. Department of Geodetic Science, The Ohio State University, OSU Report No. 148, Columbus, Ohio.
Dermanis A (1985) Optimization problems in geodetic networks with signals. In Optimization and design of geodetic networks (Grafarend EW and Sanso F, eds), Springer-Verlag, Berlin Heidelberg, pp. 221-256.

Grafarend EW (1974) Optimization of geodetic networks. Boll Geod Sci Affi, vol. XXXIII, pp. 351-406.

Koch K-R (1999) Parameter estimation and hypothesis testing in linear models, $2^{\text {nd }}$ edition. Springer-Verlag, Berlin Heidelberg.

Kotsakis C (2012) Reference frame stability and nonlinear distortion in minimum-constrained network adjustment. J Geod, 86(9): 755-774.

Kotsakis C (2013) Generalized inner constraints for geodetic network densification problems. J Geod, 87(7): 661-673.

Meissl P (1969) Zusammengfassung und Ausbau der inneren Fehlertheoric eines Punkthaufens. Deutsche Geodätische Kommission, Reihe A, 61: 8-21.

Schaffrin B (1985) Aspects of network design. In Optimization and design of geodetic networks (Grafarend EW and Sanso F, eds), SpringerVerlag, Berlin Heidelberg, pp. 549-597.

Schmitt G (1982) Optimization of geodetic networks. Rev Geoph Spac Phys, 20(4): 877-884.

Sillard P, Boucher C (2001) A review of algebraic constraints in terrestrial reference frame datum definition. J Geod, 75: 63-73.

Teunissen P (1985) Zero order design: generalized inverse, adjustment, the datum problem and $\mathrm{S}$ transformations. In Optimization and design of geodetic networks (Grafarend EW and Sanso F, eds), Springer-Verlag, Berlin Heidelberg, pp. 1155. 